\title{
Self-perceived Oral Health, Satisfaction with Overall Health and Quality of Life Comparisons between Patients with Oral Lichen Planus and their Matched Controls
}

\author{
Jolanta Aleksejuniene ${ }^{1}$, Arunas Rimkevicius ${ }^{2}$, Alina Puriene ${ }^{3}$, Ruta Rasteniene ${ }^{4}$
}

\begin{abstract}
Background: Oral lichen planus (OLP) is one of the most prevalent oral mucosal diseases.

Aims: The aim of this study is to compare the quality of life, self-perceived oral health, satisfaction with health, and their potential predictors in OLP patients and their matched controls.

Materials and methods: The study included 132 cases and 133 controls matched to cases by age, gender, and urbanization. The information about disease-related outcomes and predictors was acquired from a structured questionnaire and supplemented with interviews.

Results: OLP patients had worse self-perceived oral health and lower quality of life and were less satisfied with their health as compared to their matched controls. The worse self-reported oral health was predicted by having OLP $(O R=3.9)$, oral disease's negative impact on daily life $(\mathrm{OR}=3.0)$, and disease's impediment to eating $(\mathrm{OR}=3.8)$. Lower satisfaction with overall health was predicted by having multiple systemic conditions $(O R=1.4)$ and reporting an oral disease's negative impact on daily life $(O R=2.6)$. The only significant predictor for dissatisfaction with the quality of life was reporting the oral disease's negative impact on daily life $(O R=2.4)$.
\end{abstract}

Conclusion: Oral disease's negative impact on daily life was a significant predictor for all three-study outcomes: worse self-reported oral health, lower satisfaction with overall health, and lower quality of life.

Keywords: General health, Oral lichen planus, Quality of life, Self-reported oral health.

Journal of Contemporary Dentistry (2019): 10.5005/jp-journals-10031-1248

\section{INTRODUCTION}

Oral lichen planus (OLP), the mucosal counterpart of cutaneous lichen planus, is a T-cell-mediated autoimmune chronic inflammatory disease that affects more females than males. ${ }^{1}$ OLP commonly involves multiple sites ${ }^{2}$ and presents with several clinical manifestations and symptoms. ${ }^{3}$ OLP is one of the most common oral mucosal diseases and it affects an estimated $0.5-4.0 \%$ of the general population. ${ }^{4}$ The OLP disease most frequently affects middle-aged females. ${ }^{5}$ Around half of OLP patients report systemic diseases. ${ }^{6}$ Stress has been noted as one of the most important factors aggravating the disease. ${ }^{2}$ Some foods and dental procedures have been linked with the exacerbation of OLP. ${ }^{7}$ OLP subjects also showed a higher sensitivity to dental materials than a control population. ${ }^{8-10}$ Some studies addressed the relationship between OLP and medication use, ${ }^{11}$ while others did not associate the use of medications with the occurrence of OLP lesions. ${ }^{12}$ It has also been suggested that there are still unknown etiological factors for OLP. ${ }^{13}$

Many treatment modalities from topical corticosteroids to laser ablation of lesions have been used to treat OLP lesions. ${ }^{1}$ However, a systematic review including 28 trials compared a wide range of treatments for OLP and concluded that there is insufficient evidence to support the superior effectiveness of any specific OLP treatment. ${ }^{14}$ Concomitantly, it is important to consider that the use of drugs may be accompanied by several side effects, ${ }^{15}$ consequently, alternative medicine approaches (nonpharmacological treatments) have also been tested for the alleviation of OLP symptoms. Topical aloe vera, pimecrolimus, and oral curcuminoids seem to be the most promising of the new alternative and safe (i.e., no side effects) treatment modalities for OLP patients. ${ }^{16,17}$ General and oral quality of life is affected by oral mucosal diseases. ${ }^{18}$
${ }^{1}$ Faculty of Dentistry, University of British Columbia, Vancouver, Canada ${ }^{2-4}$ Institute of Odontology, University of Vilnius, Faculty of Medicine, Vilnius, Lithuania

Corresponding Author: Jolanta Aleksejuniene, Faculty of Dentistry, University of British Columbia, Vancouver, Canada, Phone: +370 67217824. e-mail: rasteniene.ruta@gmail.com

How to cite this article: Aleksejuniene J, Rimkevicius A, et al. Selfperceived Oral Health, Satisfaction with Overall Health and Quality of Life Comparisons between Patients with Oral Lichen Planus and their Matched Controls. J Contemp Dent 2019;9(1):1-7.

Source of support: Nil

Conflict of interest: None

These diseases can cause discomfort or spontaneous pain during eating and oral self-care. ${ }^{19}$ Disease-related psychological consequences and their impairment of social relations negatively impact a patient's everyday life. Thus, patient-centered measures should be considered for understanding the outcomes of this disease and its management. ${ }^{20}$ Patient-centered outcome measurements have been tested in OLP patients and their validity and reliability have been demonstrated. ${ }^{21}$ Such self-reported patient-centered data may provide a detailed picture of the impact of oral diseases on patients' everyday lives and life quality as well as patient-centered information may be useful in clinical practice. ${ }^{22}$

Although there are many studies with OLP patients, these studies seemed to be mainly focusing on different patient management approaches. Consequently, comprehensive studies that test multiple potential determinants that may explain or predict different patient 
outcomes are still needed. In addition, OLP manifests clinically similarly in different subjects, but multiple determinants of this disease and, particularly, self-perceptions of different oral healthrelated quality of life (OHQL) aspects might vary among individuals as well as among patients from different countries.

Therefore, the aim of the present study is to compare the quality of life, self-perceived oral health, satisfaction with overall health, and their potential predictors in OLP patients and their matched controls.

\section{Materials and Methods}

Given OLP disease is a rare disease, the case-control study design was chosen. The study was approved by the National Research Ethics Board (No. 158200-02-148-056LP11).

Data was collected during the time period of 2010-2014.

\section{Selection of Cases and Controls}

The Vilnius University's Dental Clinic consults and treats around $80-90 \%$ of the country's patients with a lichen planus diagnosis (National Health Statistics Data, 2014). These patients are referred to this clinic from different locations around the country. All patients during the time period of 2010-2013 with a histologically confirmed OLP diagnosis agreed to participate in the present study. Controls were patients not having OLP and they were recruited from a patient pool attending the same university clinic during the same time frame. The cases and controls were matched by gender, age, and urbanization. The success of matching was assessed by a Chi-square test (Table 1). There were no statistically significant proportional differences regarding age, gender, or urbanization between the cases and controls. Thus, matching cases and controls were considered satisfactory.

The final sample included a total of 266 patients, of which, 133 were cases and 133 were matched controls, who were all treated and consulted for different health conditions in the same University Hospital.

\section{Questionnaire/Interview}

Information about self-reported oral health, different aspects of general health, and oral disease-related problems was collected. Although the previously validated "the Chronic Oral Mucosal Diseases

Table 1: Demographic characteristics of cases (OLP) and their matched controls

\begin{tabular}{|c|c|c|c|c|c|}
\hline & \multicolumn{2}{|c|}{ Controls, $n(\%)$} & \multicolumn{2}{|c|}{ Cases, $n(\%)$} & Total, $n(\%)$ \\
\hline \multicolumn{6}{|c|}{ Matching by gender: Chi-squared test, $p=0.500$} \\
\hline Males & \multicolumn{2}{|c|}{$25(18.8)$} & \multicolumn{2}{|c|}{$24(18.0)$} & $49(18.4)$ \\
\hline Females & \multicolumn{2}{|c|}{$108(81.2)$} & \multicolumn{2}{|c|}{109 (82.0) } & $217(81.6)$ \\
\hline \multicolumn{6}{|c|}{ Matching by age group: Chi-squared test, $p=0.957$} \\
\hline$\leq 30$ years & \multicolumn{2}{|c|}{$12(9.0)$} & \multicolumn{2}{|c|}{$12(9.0)$} & $24(9.0)$ \\
\hline $31-40$ years & \multicolumn{2}{|c|}{$12(9.0)$} & \multicolumn{2}{|c|}{$9(6.8)$} & $21(7.9)$ \\
\hline $41-50$ years & \multicolumn{2}{|c|}{$24(18.0)$} & \multicolumn{2}{|c|}{$21(15.8)$} & $45(16.9)$ \\
\hline $51-60$ years & \multicolumn{2}{|c|}{$34(25.6)$} & \multicolumn{2}{|c|}{$38(28.6)$} & $72(27.1)$ \\
\hline $61-70$ years & \multicolumn{2}{|c|}{$30(22.6)$} & \multicolumn{2}{|c|}{$29(21.8)$} & $59(22.2)$ \\
\hline$>70$ years & \multicolumn{2}{|c|}{$21(15.8)$} & 24 & 18.0 & $45(16.9)$ \\
\hline \multicolumn{6}{|c|}{ Matching by urbanization: Chi-squared test, $p=0.769$} \\
\hline Urban & 104 & 78.2 & 102 & 76.7 & 206 (77.4) \\
\hline Semiurban/rural & 29 & 21.7 & 31 & 23.3 & $60(22.6)$ \\
\hline Total & 133 & 100.0 & 133 & 100.0 & $266(100.0)$ \\
\hline
\end{tabular}

Questionnaire"23 could be most appropriate to examine different qualities of life (QoL)-related aspects in OLP patients, this questionnaire was not appropriate for our sample of matched controls. Therefore, we decided to choose a QoL that has a more broad application.

A number of different life quality-related aspects were collected by a questionnaire (WHOQoL-100) http://www.who.int/mental_ health/who_qol_field_trial_1995.pdf.

Guidelines for across country adaptation of survey tools recommend several strategies such as back-translation technique, evaluation, and subsequent validation by experts in a particular medical field or review by independent bilingual people. ${ }^{24}$ In the present study, we translated the WHOQoL-100 questionnaire employing the forward-backward technique by two independent bilingual medical professionals.

To reduce the number of missing answers, personal interviews were added when questionnaires were incomplete. The study included three self-reported outcomes: (1) self-reported oral health status, (2) satisfaction with overall health, and (3) satisfaction with the quality of life. Information acquired through the WHOQoL-100 questionnaire about oral disease-related problems was grouped into five specific domains, each of them represented by several indicators (questions) measured on a five-item response scale (Table 2, first column). The first domain "feeling pain or discomfort due to oral disease" included eight indicators, where higher scores denote a worse health condition. Similarly, the second domain "need for medications and medical care to support normal everyday living" comprised three indicators that inquired about different health care-related aspects. The third domain inquired about the impact of oral disease on everyday living and composed of eight indicators. The fourth domain collected information about sensitivity to oral care products and oral self-care practice and this domain consisted of four indicators. The fifth domain had four questions related to oral disease as an impediment to eating.

In preparation for the multivariate analyses (binary logistic regression), a total of the worst condition scores in each of the oral disease-related problem domains were summed. These summative worst condition scores, one for each of the five oral problems-related domains, were subsequently compared between cases and controls. In addition, the following summative general health-related scores were calculated: a total number of systemic diseases (concept: multiple systemic conditions), a total number of allergies (concept: multiple allergies), and a total number of negative life events. This way, a total of nine predictors (five for oral healthrelated problems and four for general health-related problems) were prepared for the multivariate binary logistic analyses: group affiliation (OLP vs control), number of systemic conditions, number of negative life events, regarding pain or discomfort due to oral disease (worst scores in domain 1), need for medications and medical care to support everyday living (worst scores in domain 2), oral disease impact on daily life (worst scores in domain 3), sensitivity to oral self-care products and oral self-care practice (worst scores in domain 4), and oral disease-related impediment to eating (worst scores in domain 5).

\section{Statistical Analyses}

All statistical analyses were performed employing the IBM SPSS Version 21.0 statistical software. Bivariate analyses were used to evaluate differences between the cases and controls regarding the three study outcomes: (1) self-reported oral health, (2) satisfaction with overall health, and (3) satisfaction with the quality of life. The independent sample $t$ test was used to compare cases and controls 
Table 2: Disease-related problems in OLP patients and their matched controls ${ }^{\mathrm{a}}$

\begin{tabular}{|c|c|c|c|c|}
\hline \multirow[b]{3}{*}{ Disease-related domains and their indicators ${ }^{b}$} & \multicolumn{2}{|c|}{ Study groups } & \multirow[b]{3}{*}{ pvalue } & \multirow[b]{3}{*}{$95 \% \mathrm{Cl}$} \\
\hline & Cases & Controls & & \\
\hline & Mean $\pm S D$ & Mean $\pm S D$ & & \\
\hline \multicolumn{5}{|c|}{ Domain 1: feeling oral disease-related pain or discomfort } \\
\hline Feeling oral pain or discomfort & $3.0 \pm 1.1$ & $2.1 \pm 1.0$ & $<0.001$ & $-1.2 ;-0.7$ \\
\hline Difficult to tolerate oral pain & $2.6 \pm 1.0$ & $2.2 \pm 1.0$ & 0.009 & $-0.6 ;-0.1$ \\
\hline Difficult to tolerate oral discomfort & $2.7 \pm 1.0$ & $2.3 \pm 1.0$ & 0.003 & $-0.6 ;-0.1$ \\
\hline Oral pain limits daily activities & $2.3 \pm 1.2$ & $1.9 \pm 1.1$ & 0.005 & $-0.7 ;-0.1$ \\
\hline Frequent episodes of oral pain & $2.4 \pm 1.1$ & $1.6 \pm 0.6$ & $<0.001$ & $-1.0 ;-0.6$ \\
\hline Oral mucosa-related complaints & $3.0 \pm 1.0$ & $1.6 \pm 0.7$ & $<0.001$ & $-1.6 ;-1.2$ \\
\hline \multicolumn{5}{|c|}{ Domain 2: need for medications and medical care due to oral disease } \\
\hline Need for medications & $1.8 \pm 0.9$ & $1.5 \pm 0.7$ & 0.006 & $-0.5 ;-0.1$ \\
\hline Need for regular medical care & $2.5 \pm 1.1$ & $1.7 \pm 1.0$ & $<0.001$ & $-1.0 ;-0.5$ \\
\hline Need for regular treatment and medications & $1.8 \pm 1.1$ & $1.5 \pm 0.9$ & 0.015 & $-0.5 ;-0.1$ \\
\hline \multicolumn{5}{|l|}{ Domain 3: oral disease's negative impact on daily life } \\
\hline Disease-related future concern & $2.3 \pm 0.9$ & $1.4 \pm 0.8$ & $<0.001$ & $0.7 ; 1.1$ \\
\hline Disease impact on everyday living & $2.3 \pm 1.0$ & $1.8 \pm 1.0$ & $<0.001$ & $-0.8 ;-0.3$ \\
\hline Daily concern about disease & $2.9 \pm 1.1$ & $2.0 \pm 1.0$ & $<0.001$ & $-1.2 ;-0.6$ \\
\hline Disease-related anxiety limiting daily work & $1.8 \pm 1.0$ & $1.4 \pm 0.7$ & $<0.001$ & $-0.6 ;-0.2$ \\
\hline Having moods due to oral disease & $1.7 \pm 0.9$ & $1.4 \pm 0.7$ & 0.003 & $-0.5 ;-0.1$ \\
\hline Disease limiting enjoyment of leisure time & $1.8 \pm 1.0$ & $1.5 \pm 0.8$ & 0.004 & $-0.5 ;-0.1$ \\
\hline Disease allowing relaxation & $3.4 \pm 1.2$ & $4.0 \pm 1.0$ & $<0.001$ & $0.4 ; 0.9$ \\
\hline \multicolumn{5}{|c|}{ Domain 4: sensitivity to oral self-care products and practice } \\
\hline Ability to use oral self-care products & $2.9 \pm 1.4$ & $3.8 \pm 1.2$ & $<0.001$ & $0.6 ;-1.2$ \\
\hline Toothpastes evoke unpleasant sensations & $2.2 \pm 1.2$ & $1.5 \pm 0.7$ & $<0.001$ & $-0.9 ;-0.4$ \\
\hline Mouth rinses evoke unpleasant sensations & $1.9 \pm 1.2$ & $1.6 \pm 0.7$ & 0.015 & $-0.5 ;-0.1$ \\
\hline Lesions cause discomfort during oral self-care & $2.9 \pm 1.1$ & $1.4 \pm 0.8$ & $<0.001$ & $-1.7 ;-1.2$ \\
\hline \multicolumn{5}{|l|}{ Domain 5: oral disease-related impediment to eating } \\
\hline Discomfort while chewing hard food & $2.6 \pm 1.3$ & $1.8 \pm 0.9$ & $<0.001$ & $-1.0 ;-0.5$ \\
\hline Acidic foods provoke pain & $2.7 \pm 1.4$ & $1.6 \pm 0.7$ & $<0.001$ & $-1.0 ;-0.5$ \\
\hline Spicy foods provoke pain & $2.9 \pm 1.4$ & $1.5 \pm 0.8$ & $<0.001$ & $-1.6 ;-1.1$ \\
\hline Unable to consume some food items & $2.4 \pm 1.2$ & $1.6 \pm 0.9$ & $<0.001$ & $-1.0 ;-0.6$ \\
\hline
\end{tabular}

andependent sample $t$ test

"Indicators structured on a five-item response scale (from " 1 " being the lowest to " 5 " being the highest)

regarding the following predictors: the mean numbers of systemic conditions, allergies, and negative life events and to compare the two patient groups regarding the five domains of oral diseaserelated problems: (1) sensitivity to oral self-care products, (2) need for medications and medical care, (3) feeling in pain or discomfort due to oral disease, (4) oral disease impact on daily life, and (5) oral disease-related impediment to eating.

Three multivariate logistic regression models were tested (Table 4). Model 1 had a binary outcome "self-reported oral health" and it compared patients perceiving their oral health as "very good" or "good" vs the ones who perceived their oral health as "average," "poor," or "very poor." Model 2 had a binary outcome "satisfaction with overall health" and it compared the ones who were very satisfied or satisfied with their overall health with the ones who were either neither satisfied or dissatisfied, dissatisfied or very dissatisfied. Model's 3 binary outcome was "overall satisfaction with the quality of life" and compared the patients who were very satisfied or satisfied with the ones who were neutral, dissatisfied or very dissatisfied.

Models 1 and 2 tested a total of nine predictors (Table 4): (1) group affiliation (control condition vs OLP), (2) a total number of systemic conditions, (3) a total number of allergies, (4) a total number of negative life events, (5) oral disease's negative impact on daily life, (6) sensitivity to oral care products or oral self-care practice, (7) need for regular medications and care, (8) feeling oral disease pain or discomfort, and (9) oral disease's impediment to eating. Model 3 included also an additional predictor, namely self-perceived oral health.

The -2 log likelihood was used as a method for the multivariate statistical testing. The goodness of a model fit (presented as summaries in Table 4) was based on the overall model's significance $(p<0.05)$ and the value of the Nagelkerke R Square statistical parameter, which denotes how much of the variance in the dependent outcome is explained by a set of predictors. The value of each predictor included in a multivariate logistic regression model is presented with a significance ( $p$ value) and odds ratio with a 95\% confidence interval $(\mathrm{Cl})$.

\section{Results}

Figures 1 to 3 illustrate the participants' distribution regarding the three study outcomes. Self-reported oral health was considerably worse among the OLP patients than among their matched controls. 


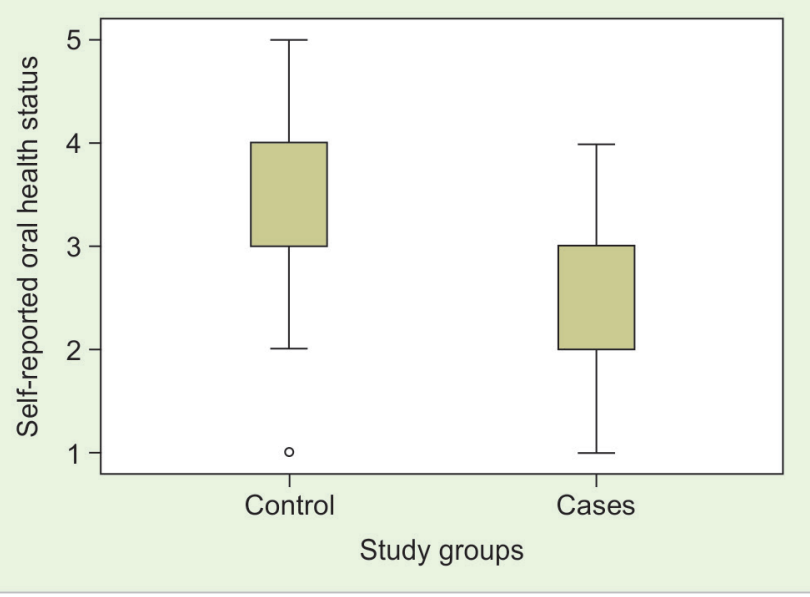

Fig. 1: Self-reported oral health status in study groups

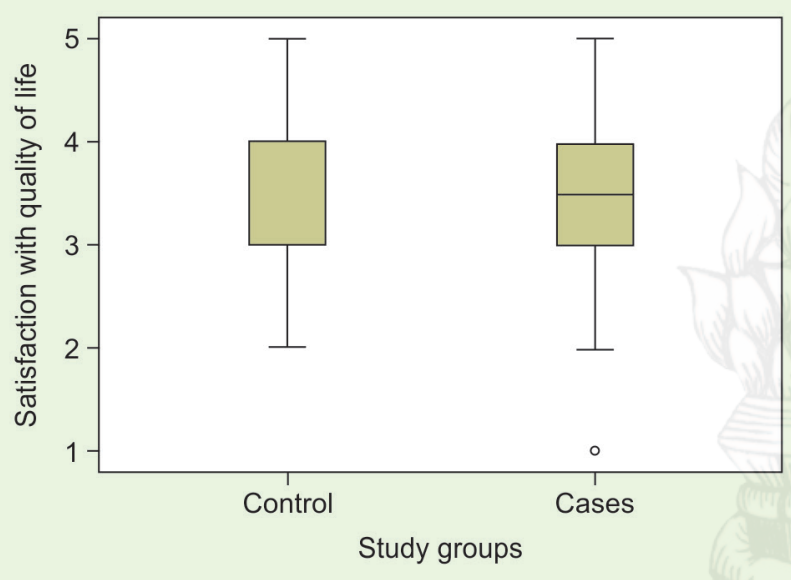

Fig. 3: Satisfaction with quality of life in lichen planus patients and matched controls

Of all studied, over $75 \%$ of OLP patients perceived their oral health as average or worse and $75 \%$ of the control group patients perceived their oral health to be average or better.

Figure 1 shows patient distribution regarding their selfreported oral health in two study groups. One can see that more patients with worse self-reported oral health were in the OLP group than in the control group.

Figure 2 visually compares participant satisfaction with their overall health. Two trends can be observed. First, there was considerably less variation in the control group as compared to the OLP patient group. Second, similar to self-reported oral health, satisfaction with general health was lower in the group of OLP cases as compared with the group of controls.

Figure 3 compares the distribution of cases and controls regarding satisfaction with the quality of life. Similar trends, as in the other two study outcomes, could be observed; for example, more OLP patients were less satisfied with the quality of life as compared to their age, gender, and residency-matched controls.

In Table 2, the mean scores regarding a number of oral diseaserelated problems in five domains are compared between the OLP patients and their matched controls. There were statistically significant differences between the two study groups in all five oral disease-related problem domains. For example, there was

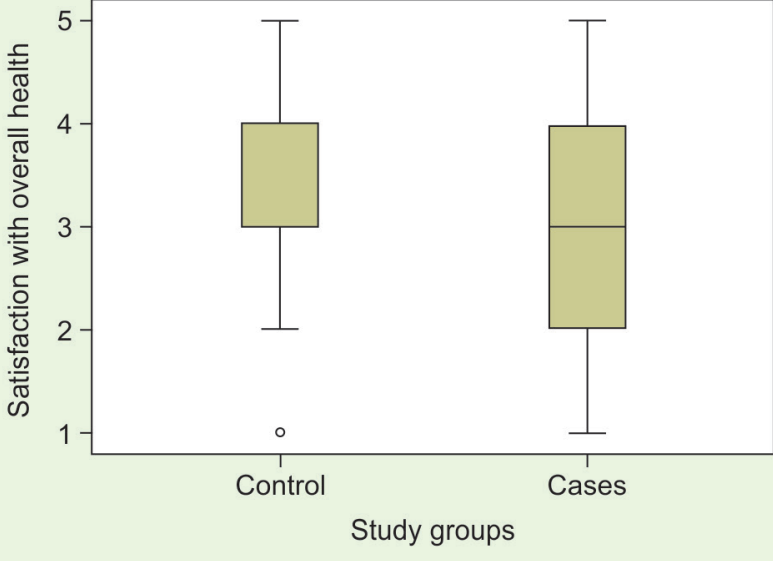

Fig. 2: Satisfaction with overall health in lichen planus patients and matched controls

statistically significantly more oral pain or discomfort in the OLP group as compared with the control group. When examining the domain 1 indicators, the most pronounced differences between the two study groups related to oral mucosal complaints was that OLP patients reported such complaints more frequently than patients from the control group.

The domain 2 indicators provided information about a need for regular medication and medical care to support normal everyday living. There were some patients in both groups who needed regular medications and medical care, but statistically significantly more of such patients were in the OLP group.

When examining domain 3 indicators, a significant difference was observed between the cases and controls regarding future disease-related concerns, oral disease impact on daily life, or disease-related psychological impact. When examining domain 4 indicators, a consistent trend was noticed where OLP patients had difficulties using oral self-care products. Similar OLP-related impediments were observed in domain 5 where OLP patients, as compared to patients without OLP, more frequently had problems consuming acidic and spicy foods or experienced discomfort due to chewing hard food items.

Table 3 presents results where OLP cases are compared to their matched controls regarding the three dependent outcomes: (1) selfreported oral health, (2) overall satisfaction with general health, and (3) overall satisfaction with the quality of life. Statistically significant differences between OLP cases and their demographically matched controls were found in all three study outcomes. More OLP patients had multiple systemic conditions and allergies, while there was no significant difference between the study groups in experiencing negative life events.

To acquire an overall trend of the deterioration due to oral diseases, summative worst condition scores within each domain of oral disease-related problems were calculated. Examining the summative worst conditions scores showed that OLP cases as compared to controls had significantly more severe oral diseaserelated pain or discomfort, needed regular medications and medical care more frequently, suffered from oral disease daily, and had problems using oral self-care products and eating varying food items.

For further multivariate analyses, the three study outcomes were dichotomized and multiple predictors were tested in binary logistic analyses. Table 4 presents the results of this testing with 
Table 3: Self-reported oral health, satisfaction with general health and satisfaction with the quality of life in OLP patients and their matched controls ${ }^{\mathrm{a}}$

\begin{tabular}{|c|c|c|c|c|}
\hline & \multicolumn{2}{|c|}{ Groups } & \multirow[b]{3}{*}{$p$ values } & \multirow[b]{3}{*}{$95 \% \mathrm{Cl}$} \\
\hline & Cases & Controls & & \\
\hline & Mean $\pm S D$ & Mean $\pm S D$ & & \\
\hline \multicolumn{5}{|c|}{ Satisfaction with health and quality of life (outcomes) } \\
\hline Self-reported oral health & $2.6 \pm 0.8$ & $3.4 \pm 0.9$ & 0.001 & $0.7 ; 1.1$ \\
\hline Satisfaction with overall health & $3.0 \pm 0.9$ & $3.5 \pm 0.8$ & $<0.001$ & $0.3 ; 0.7$ \\
\hline Satisfaction with the quality of life & $3.6 \pm 0.8$ & $3.8 \pm 0.8$ & 0.031 & $0.2 ; 0.4$ \\
\hline \multicolumn{5}{|c|}{ General health-related summative scores (predictors) } \\
\hline Multiple systemic conditions & $2.8 \pm 2.4$ & $1.5 \pm 1.6$ & $<0.001$ & $-1.8 ;-0.9$ \\
\hline Multiple allergies & $0.5 \pm 0.9$ & $0.2 \pm 0.5$ & $<0.001$ & $-0.5 ;-0.2$ \\
\hline Multiple negative life events & $1.6 \pm 1.2$ & $1.8 \pm 1.6$ & 0.141 & $-0.1 ; 0.6$ \\
\hline \multicolumn{5}{|c|}{ Domain-based summative worst conditions scores (predictors) } \\
\hline Pain or discomfort due to oral disease & $1.4 \pm 1.7$ & $0.4 \pm 0.9$ & $<0.001$ & $-1.3 ;-0.6$ \\
\hline Need for medications and care & $0.5 \pm 0.9$ & $0.2 \pm 0.6$ & 0.020 & $-0.4 ;-0.1$ \\
\hline Oral disease impact on daily life & $1.3 \pm 1.7$ & $0.5 \pm 0.9$ & $<0.001$ & $-1.2 ;-0.5$ \\
\hline Sensitivity to oral self-care products & $1.0 \pm 1.2$ & $0.3 \pm 0.5$ & $<0.001$ & $-0.9 ;-0.5$ \\
\hline Oral disease impediments to eating & $1.3 \pm 1.5$ & $0.2 \pm 0.5$ & $<0.001$ & $-1.4 ;-0.9$ \\
\hline
\end{tabular}

three binary outcome variables. The good or very good selfreported oral health was tested vs average or poor or very poor self-reported oral health. Similarly, the satisfaction with overall health (very satisfied/satisfied) was tested vs the ones who were neither satisfied or were dissatisfied/very dissatisfied with overall health. Similarly, answers regarding the satisfaction with the quality of life were grouped (very satisfied/satisfied vs neither satisfied or were dissatisfied/very dissatisfied).

The binary logistic regression model for the self-reported health included nine predictors and the overall model was statistically significant $(p=0.002)$. The strongest predictor of worse selfreported oral health was the group affiliation $(\mathrm{OR}=3.9)$ indicating that OLP patients statistically significantly reported their oral health to be worse even after controlling for other predictors. Other significant predictors of worse oral health were the following: (i) oral disease having a negative impact on daily living $(O R=3.0)$ and (ii) having problems when consuming acidic, spicy, and hard food items $(O R=3.8)$.

The second regression model for the satisfaction with overall health was also highly statistically significant $(p<0.001)$ and significant predictors of this outcome were the following: (i) oral disease's negative impact on everyday life $(\mathrm{OR}=2.6)$ and (ii) presence of multiple systemic conditions $(O R=1.4)$.

The third logistic regression model for the satisfaction with the quality of life was highly significant $(p<0.001)$ and the only significant predictor in this model was an oral disease-related negative impact on everyday life $(\mathrm{OR}=2.4)$.

\section{Discussion}

The present 4-year prospective study examined and compared two patient groups: (1) a group of consecutive patients with validated histologically OLP diagnosis (cases) and (2) a group of age, gender, and residency-matched patients without OLP (controls). All our study outcomes - self-reported oral health, satisfaction with overall health, and satisfaction with the quality of life-were statistically significantly worse in patients with OLP when compared to similar patients not having OLP. Anxiety and depression in patients with OLP and a negative impact of the disorder on their quality of life have been previously. ${ }^{25,26}$ Similar findings to the present study that both general and oral health were impaired in patients with oral mucosal diseases have been reported elsewhere. ${ }^{6}$ Diabetes, hypertension, hepatitis $C$, and thyroiditis have been the most frequently associated systemic diseases with OLP. ${ }^{27}$ Our findings are also in accordance with the Pippi et al. case-control study which reported significant associations among smoking, psychological factors, and OLP. ${ }^{28}$

In the present study, OLP patients as compared to their matched controls more frequently experienced oral disease-related pain or discomfort. Our OLP patients also more frequently needed medications and medical care to support their everyday life. Most importantly, oral disease-related negative impact on daily life was a significant predictor for all three study outcomes, namely that of worse self-reported oral health, less satisfaction with general health, and less satisfaction with the quality of life. The other important significant predictors for worse oral health were the group affiliation (OLP vs control) and oral disease-related impediments to eating. Interestingly, the experience of negative life events was not significantly associated with either lower satisfaction with overall health or with lower satisfaction with the quality of life. This finding is in contrast to earlier studies where stress was identified as an important factor for the aggravation of OLP disease. ${ }^{29}$ It is important to consider that stressful negative life events may influence people differently, i.e., people cope with stress in different ways. Therefore, future studies are needed to assess whether different strategies for coping with stress and the presence of social support networks have positive effects on patients suffering from OLP.

The significant predictors of dissatisfaction with overall health were the following: (i) presence of multiple systemic conditions and (ii) oral disease's negative impact on life. The latter was the only significant predictor of worse overall quality of life when it was controlled for several other predictors such as having OLP disease, suffering from multiple systemic conditions, multiple allergies, experiencing multiple negative life events, or having 
Satisfaction with Overall Health and Quality of Life with OLP

Table 4: Predictors of health and quality of life-related outcomes (Binary Logistic Regression)

\begin{tabular}{|c|c|c|c|}
\hline \multicolumn{4}{|c|}{ Model: $(n=265)$ : very good/good $(n=195)$ vs average/poor/very poor $(n=70)$} \\
\hline \multicolumn{4}{|c|}{ Model summary: method -2 log likelihood $=196, p<0.001$, Nagelkerke $R$ square $=0.497$} \\
\hline Predictors & p value & Odds ratio & $95 \% \mathrm{Cl}$ \\
\hline Group affiliation $^{\mathrm{a}}$ & 0.002 & 3.9 & $1.6 ; 9.5$ \\
\hline Number of systemic conditions & 0.337 & 1.1 & $0.9 ; 1.3$ \\
\hline Number of allergies & 0.399 & 0.8 & $0.5 ; 1.3$ \\
\hline Number of negative life events & 0.973 & 1.0 & $0.4 ; 7.1$ \\
\hline Oral disease's negative impact on daily life & 0.007 & 3.0 & $1.3 ; 6.8$ \\
\hline Sensitivity to oral care products and practice & 0.258 & 1.6 & $0.7 ; 3.5$ \\
\hline Need for regular medications and care & 0.473 & 0.4 & $0.6 ; 3.4$ \\
\hline Feeling oral disease pain or discomfort & 0.088 & 2.1 & $0.9 ; 4.9$ \\
\hline Oral disease's impediments to eating & 0.001 & 3.8 & $1.7 ; 8.6$ \\
\hline
\end{tabular}

Outcome 2: satisfaction with overall health. Predictor selection: enter

\begin{tabular}{|c|c|c|c|}
\hline \multicolumn{4}{|c|}{ Model summary: method -2 log likelihood $=293, p<0.001$, Nagelkerke $R$ square $=0.311$} \\
\hline Predictors & pvalue & Odds ratio & $95 \% \mathrm{Cl}$ \\
\hline Group affiliation $^{a}$ & 0.648 & 1.2 & $0.6 ; 2.2$ \\
\hline Number of systemic conditions & 0.001 & 1.4 & $1.1 ; 1.6$ \\
\hline Number of allergies & 0.084 & 1.6 & $0.9 ; 2.6$ \\
\hline Number of negative life events & 0.154 & 1.2 & $1.0 ; 1.4$ \\
\hline Oral disease's negative impact on daily life & 0.006 & 2.6 & $1.3 ; 5.1$ \\
\hline Sensitivity to oral care products and practice & 0.720 & 1.1 & $0.6 ; 2.2$ \\
\hline Need for regular medications and care & 0.074 & 2.2 & $0.9 ; 5.0$ \\
\hline Feeling oral disease pain or discomfort & 0.378 & 0.7 & $0.3 ; 1.5$ \\
\hline Oral disease's impediments to eating & 0.077 & 2.0 & $0.9 ; 4.5$ \\
\hline
\end{tabular}

Outcome 3: overall satisfaction with the quality of life. Predictor selection: enter

\begin{tabular}{|c|c|c|c|}
\hline \multicolumn{4}{|c|}{ Model summary: method -2 log likelihood $=303, p<0.001$, Nagelkerke $R$ square $=0.177$} \\
\hline Predictors & $p$ value & Odds ratio & $95 \% \mathrm{Cl}$ \\
\hline Group affiliation $^{a}$ & 0.665 & 1.2 & $0.6 ; 2.3$ \\
\hline Self-reported oral health & 0.103 & 1.4 & $0.9 ; 2.1$ \\
\hline Number of systemic conditions & 0.100 & 0.9 & $0.8 ; 1.0$ \\
\hline Number of allergies & 0.262 & 0.8 & $0.7 ; 1.2$ \\
\hline Number of negative life events & 0.223 & 0.9 & $0.8 ; 1.1$ \\
\hline Oral disease's negative impact on daily life & 0.009 & 2.4 & $1.3 ; 4.7$ \\
\hline Sensitivity to oral care products and practice & 0.337 & 1.4 & $0.8 ; 2.7$ \\
\hline Need for regular medications and care & 0.760 & 0.9 & $0.4 ; 1.9$ \\
\hline Feeling oral disease pain or discomfort & 0.626 & 1.2 & $0.6 ; 2.5$ \\
\hline Oral disease's impediments to eating & 0.983 & 1.0 & $0.5 ; 2.2$ \\
\hline
\end{tabular}

${ }^{a}$ Group affiliation control condition vs OLP

oral disease-related problems such as being in need of regular medications or medical care or not being able to eat varying foods or having problems in practicing oral self-care.

Some limitations of the present study need to be discussed. Although OLP is one of the most clinically common mucocutaneous diseases, due to its relatively low prevalence $(0.2-4.0 \%),{ }^{3}$ it can be considered a rare disease. Therefore, we chose a case-control study design which is considered an optimal and practical study design for studying rare diseases. However, this study design has limitations as it does not allow causal interpretations of study findings. Consequently, all significant differences we found between OLP patients and their matched controls could only be interpreted as associations. Another deficiency of the current work is a relatively small sample size in each of the two study groups ( $n=133)$; this limitation precluded us from performing more specific subgroup analyses.

Similar to recent reports, ${ }^{22}$ our OLP patients had an overall lower quality of life potentially due to their oral disease even when it was controlled for systemic conditions. This significant finding means that OLP-related impediments to everyday life may 
be a supplemental burden to patients who already suffer from other systemic diseases. Concomitantly, we need to consider our limitation that we did not use the questionnaire (Chronic Oral Mucosal Diseases Questionnaire) that was specifically validated for the use in samples of patients suffering from chronic oral mucosal diseases. ${ }^{23}$ Possibly, the use of such measure might have helped to identify additional aspects of reduced quality of life. Another limitation of the present work is that it applied across-countryvalidated WHO recommended measurement of $\mathrm{OHQL}$; however, we used the translated questionnaire, but none of the formal validation of this translated OHQL tool was employed.

More efficient OLP treatment protocols should be developed to strive toward the goal of improvement in the everyday lives of OLP patients as well as maintaining their quality of life.

\section{Conclusions}

Patients with OLP as compared to their gender, age, and residencymatched controls without OLP presented worse self-reported oral health, less satisfaction with their overall health, and less satisfaction with their quality of life. Worse self-reported oral health was predicted by having OLP through the oral disease's negative impact on daily life and the oral disease's impediments to eating. Low satisfaction with overall health was predicted by the presence of multiple systemic conditions in addition to the oral disease's negative impact on life. The only significant predictor for lower satisfaction with the quality of life was OLP's negative impact on daily life.

\section{ACKNOWLedgment}

The authors would like to thank Ms Claire Davis University of British Columbia for editorial assistance.

\section{References}

1. Lavanya $\mathrm{N}$, Jayanthi $\mathrm{P}$, et al. Oral lichen planus: an update on pathogenesis and treatment. J Oral Maxillofac Pathol 2011;15(2): 127-132. DOI: 10.4103/0973-029X.84474.

2. Bajaj DR, Khoso NA, et al. Oral lichen planus: a clinical study. J Coll Physicians Surg Pak 2010;20(3):154-157. DOI: 03.2010/JCPSP.154157.

3. Au J, Patel $D$, et al. Oral lichen planus. Oral Maxillofac Surg Clin North Am 2013;25(1):93-100, vii. DOI: 10.1016/j.coms.2012.11.007.

4. McCartan $\mathrm{BE}$, Healy $\mathrm{CM}$. The reported prevalence of oral lichen planus: a review and critique. J Oral Pathol Med 2008;37:447-453.

5. Ismail SB, Kumar SK, et al. Oral lichen planus and lichenoid reactions: etiopathogenesis, diagnosis, management and malignant transformation. J Oral Sci 2007;49(2):89-106. DOI: 10.2334/ josnusd.49.89.

6. Bardellini E, Amadori F, et al. Clinicopathological features and malignant transformation of oral lichen planus: a 12-years retrospective study. Acta Odontol Scand 2013;71(3-4):834-840. DOI: 10.3109/00016357.2012.734407.

7. Eisen D. The clinical features, malignant potential, and systemic associations of oral lichen planus: a study of 723 patients. J Am Acad Dermatol 2002;46(2):207-214. DOI: 10.1067/mjd.2002.120452.

8. Martin MD, Broughton S, et al. Oral lichen planus and dental materials: a case control study. Contact Dermatitis 2003;48(6):331-336. DOI: 10.1034/j.1600-0536.2003.00146.x.
9. Yiannias JA, el-Azhary RA, et al. Relevant contact sensitivities in patients with the diagnosis of oral lichen planus. J Am Acad Dermatol 2000;42(2 Pt 1):177-182. DOI: 10.1016/S0190-9622(00)90123-3.

10. Eversole LR, Ringer $M$. The role of dental restorative metals in the pathogenesis of oral lichen planus. Oral Surg Oral Med Oral Pathol 1984;57(4):383-387. DOI: 10.1016/0030-4220(84)90155-5.

11. Thompson DF, Skaehill PA. Drug-induced lichen planus. Pharmacotherapy 1994;14(5):561-571.

12. Hirota SK, Moreno RA, et al. Analysis of a possible association between oral lichen planus and drug intake. A controlled study. Med Oral Patol Oral Cir Bucal 2011;16(6):e750-e756. DOI: 10.4317/medoral.17095.

13. Boorghani $\mathrm{M}$, Gholizadeh $\mathrm{N}$, et al. Oral lichen planus: clinical features, etiology, treatment and management: a review of literature. J Dent Res Dent Clin Dent Prospects 2010;4(1):3-9. DOI: 10.5681/joddd.2010.002.

14. Lodi G, Carrozzo M, et al. Interventions for treating oral lichen planus: a systematic review. Br J Dermatol 2012;166(5):938-947. DOI: 10.1111/j.1365-2133.2012.10821.x.

15. Crincoli V, Di Bisceglie MB, et al. Oral lichen planus: update on etiopathogenesis, diagnosis and treatment. Immunopharmacol Immunotoxicol 2011;33(1):11-20. DOI: 10.3109/08923973.2010.498014.

16. Thongprasom K, Prapinjumrune $C$, et al. Novel therapies for oral lichen planus. J Oral Pathol Med 2013 May 13;42(10):721-727. DOI: 10.1111/jop.12083.

17. Choonhakarn $C$, Busaracome $\mathrm{P}$, et al. The efficacy of aloe vera gel in the treatment of oral lichen planus: a randomized controlled trial. $\mathrm{Br}$ JDermatol 2008;158(3):573-577.DOI: 10.1111/j.1365-2133.2007.08370.x.

18. Liu LJ, Xiao W, et al. Generic and oral quality of life is affected by oral mucosal diseases. BMC Oral Health 2012;12:2. DOI: 10.1186/1472-6831$12-2$.

19. Nagao Y, Sata M. Effect of oral care gel on the quality of life for oral lichen planus in patients with chronic HCV infection. Virol J 2011;8:348. DOI: 10.1186/1743-422X-8-348.

20. Lopez-Jornet $P$, Camacho-Alonso F. Quality of life in patients with oral lichen planus. J Eval Clin Pract 2010;16(1):111-113. DOI: 10.1111/j.13652753.2009.01124.x.

21. Hegarty AM, McGrath C, et al. Patient-centred outcome measures in oral medicine: are they valid and reliable? Int J Oral Maxillofac Surg 2002;31(6):670-674. DOI: 10.1054/ijom.2002.0260.

22. Lopez-Jornet $\mathrm{P}$, Camacho-Alonso F, et al. Measuring the impact of oral mucosa disease on quality of life. Eur J Dermatol 2009;19(6):603-606. DOI: 10.1684/ejd.2009.0762.

23. Riordain RN, Hodgson T, et al. Validity and reliability of the Chronic Oral Mucosal Diseases Questionnaire in a UK population. J Oral Pathol Med 2016 Jan 26;45(8):613-616. DOI: 10.1111/jop.12425.

24. Danielsen AK, Pommergaard HC, et al. Translation of Questionnaires Measuring Health Related Quality of Life is not Standardized: A literature Based Research study. PLoS One 2015;10(5):e0127050. DOI: $10.1371 /$ journal.pone.0127050.

25. Barbosa NG, Silveira EJ, et al. Factors associated with clinical characteristics and symptoms in a case series of oral lichen planus. Int J Dermatol 2015;54(1):e1-e6. DOI: 10.1111/ijd.12485.

26. Alves MG, do Carmo Carvalho BF, et al. Emotional assessment of patients with oral lichen planus. Int J Dermatol 2015;54(1):29-32. DOI: $10.1111 /$ ijd.12052.

27. Lauritano $D$, Arrica $M$, et al. Oral lichen planus clinical characteristics in Italian patients: a retrospective analysis. Head Face Med 2016;12:18. DOI: 10.1186/s13005-016-0115-z.

28. Pippi R, Romeo U, et al. Psychological disorders and oral lichen planus: matched case-control study and literature review. Oral Diseases 2016;22:226-234. DOI: 10.1111/odi.12423.

29. Carrozzo M, Thorpe R. Oral lichen planus: a review. Minerva Stomatol 2009;58(10):519-537. DOI: 10.1054/bjom.2000.0335. 